\title{
Roles of TBC1D1 and TBC1D4 in insulin- and exercise-stimulated glucose transport of skeletal muscle
}

\author{
Gregory D. Cartee
}

Received: 30 June 2014 / Accepted: 7 August 2014 / Published online: 4 October 2014

(C) Springer-Verlag Berlin Heidelberg 2014

\begin{abstract}
This review focuses on two paralogue Rab GTPase activating proteins known as TBC1D1 Tre-2/BUB2/cdc 1 domain family (TBC1D) 1 and TBC1D4 (also called Akt Substrate of $160 \mathrm{kDa}, \mathrm{AS160}$ ) and their roles in controlling skeletal muscle glucose transport in response to the independent and combined effects of insulin and exercise. Convincing evidence implicates Akt2-dependent TBC1D4 phosphorylation on T642 as a key part of the mechanism for insulinstimulated glucose uptake by skeletal muscle. TBC1D1 phosphorylation on several insulin-responsive sites (including T596, a site corresponding to T642 in TBC1D4) does not appear to be essential for in vivo insulin-stimulated glucose uptake by skeletal muscle. In vivo exercise or ex vivo contraction of muscle result in greater TBC1D1 phosphorylation on S237 that is likely to be secondary to increased AMPactivated protein kinase activity and potentially important for contraction-stimulated glucose uptake. Several studies that evaluated both normal and insulin-resistant skeletal muscle stimulated with a physiological insulin concentration after a single exercise session found that greater post-exercise insulin-stimulated glucose uptake was accompanied by greater TBC1D4 phosphorylation on several sites. In contrast, enhanced post-exercise insulin sensitivity was not accompanied by greater insulin-stimulated TBC1D1 phosphorylation. The
\end{abstract}

G. D. Cartee $(\bowtie)$

Muscle Biology Laboratory, School of Kinesiology,

University of Michigan, 401 Washtenaw Avenue, Ann Arbor, MI 48109-2214, USA

e-mail: gcartee@umich.edu

G. D. Cartee

Department of Molecular and Integrative Physiology,

University of Michigan, Ann Arbor, MI, USA

G. D. Cartee

Institute of Gerontology, University of Michigan,

Ann Arbor, MI, USA mechanism for greater TBC1D4 phosphorylation in insulinstimulated muscles after acute exercise is uncertain, and a causal link between enhanced TBC1D4 phosphorylation and increased post-exercise insulin sensitivity has yet to be established. In summary, TBC1D1 and TBC1D4 have important, but distinct roles in regulating muscle glucose transport in response to insulin and exercise.

Keywords Akt substrate of $160 \mathrm{kDa} \cdot$ Diabetes · Glucose transport $\cdot$ High-fat diet $\cdot$ Insulin resistance $\cdot$ Obesity $\cdot$ Physical activity $\cdot$ Review

\begin{tabular}{|c|c|}
\hline \multicolumn{2}{|c|}{ Abbreviations } \\
\hline AMPK & 5' AMP-activated kinase \\
\hline APPL2 & $\begin{array}{l}\text { Adaptor protein containing PH domain, } \\
\text { PTB domain and leucine zipper motif }\end{array}$ \\
\hline CaMKK $\alpha$ & $\begin{array}{l}\mathrm{Ca}^{2+} / \text { calmodulin-dependent protein } \\
\text { kinase kinase } \alpha\end{array}$ \\
\hline CBD & Calmodulin-binding domain \\
\hline EDL & Extensor digitorum longus muscle \\
\hline GAP & GTPase-activating protein \\
\hline GSK3 & Glycogen synthase kinase 3 \\
\hline LFD & Low-fat diet \\
\hline HFD & High-fat diet \\
\hline PAS & Phospho Akt substrate \\
\hline PI3K & Phosphatidylinositol 3-kinase \\
\hline PP2A & Protein phosphatase $2 \mathrm{~A}$ \\
\hline РTB & Phosphotyrosine-binding \\
\hline RSK1 & $90 \mathrm{kDa}$ ribosomal S6 kinase 1 \\
\hline SGK1 & $\begin{array}{l}\text { Serum- and glucocorticoid-responsive } \\
\text { kinase } 1\end{array}$ \\
\hline $\mathrm{S} / \mathrm{T}$ kinase & Serine/threonine kinase \\
\hline TA & Tibialis anterior muscle \\
\hline TBC1D & Tre-2/BUB2/cdc 1 domain family \\
\hline$\dot{V} \mathrm{O}_{2 \text { peak }}$ & Peak oxygen consumption \\
\hline WT & Wild type \\
\hline
\end{tabular}




\section{Introduction}

The most important physiological stimuli for increasing skeletal muscle glucose transport are insulin and exercise [1-4]. Each independently triggers the redistribution of GLUT4 transporters from the cell interior to cell surface membranes $[4,5]$. In addition, a single exercise session can induce a subsequent increase in insulin-stimulated glucose transport in muscle and improved whole body insulin sensitivity for up to $\sim 2-48 \mathrm{~h}$ post exercise [6-10]. This review focuses on two paralogue Rab-GTPase-activating proteins (Rab GAPs) that are members of the tre-2/BUB2/cdc 1 domain family (TBC1D; TBC1D1 and TBC1D4 [also known as Akt substrate of $160 \mathrm{kDa}, \mathrm{AS} 160]$ ), and their roles in regulating muscle glucose transport stimulated by insulin and/or exercise.

Early research on TBC1D1 and TBC1D4 phosphorylation used a commercially available polyclonal antibody (the phospho Akt substrate [PAS] antibody) that was created to react with phosphorylated $\mathrm{S} / \mathrm{T}$ residues in Akt phosphomotifs on many proteins to identify novel Akt substrates [11-15]. Because there are multiple potential PAS-reactive phosphomotifs on TBC1D1 and TBC1D4 (proteins separated by only $\sim 10 \mathrm{kDa}$ using SDS-PAGE), care is needed when interpreting anti-PAS bands in immunoblots. In this review, numbers identifying amino acids refer to the human protein unless otherwise specified. Anti-PAS was found to have high affinity for TBC1D4 phosphorylated on T642 (pT642TBC1D4) and lower affinity for TBC1D4 phosphorylated on S588 (pS588-TBC1D4) [11]. It also had high affinity for TBC1D1 phosphorylated on T596 [16, 17] and may recognise other TBC1D1 and TBC1D4 phosphosites. This review focuses primarily on results obtained using antibodies that identify specific TBC1D1 or TBC1D4 phosphosites, and on results acquired using anti-PAS after immunoprecipitating the sample with anti-TBC1D1 or anti-TBC1D4 (PAS-TBC1D1 or PAS-TBC1D4). Unless otherwise specified, results in human muscle refer to the vastus lateralis.

\section{TBC1D1 and TBCD4: muscle fibre type}

TBC1D4 protein abundance in mice was about tenfold greater in the soleus muscle vs extensor digitorum longus muscle (EDL) or tibialis anterior muscle (TA), and abundance of TBC1D1 in TA exceeded that in EDL (by approximately threefold) and soleus (by approximately tenfold) [18]. Mouse soleus was enriched with fibres expressing myosin heavy chain type I or IIa isoforms, with lower type IIb and IIx expression, whereas mouse TA and EDL were enriched with fibres expressing myosin heavy chain type IIb or IIx isoforms and lacked detectable type I or IIa expression. In contrast, TBC1D4 abundance did not differ among multiple rat muscles with divergent fibre type profiles, including the soleus (primarily type I), EDL and TA (primarily IIb and IIx) [19]. TBC1D1 abundance did not differ in rat soleus, EDL or TA. Significant differences were undetectable for TBC1D1 or TBC1D4 abundance in human gastrocnemius, vastus lateralis and soleus [20]. Striking differences in TBC1D1 and TBC1D4 abundance found among mouse muscles have not been observed for rats or humans. Determination of fibre type, TBC1D1 and TBC1D4 abundance in individual muscle fibres would provide insights that cannot be discerned using tissuelevel analysis.

\section{TBC1D4: insulin-stimulated GLUT4 translocation in adipocytes}

Control of GLUT4 translocation by insulin requires the binding of insulin to its receptor, leading to tyrosine phosphorylation of the receptor and then of IRSs, promoting IRS engagement with phosphatidylinositol 3-kinase, facilitating phosphorylation (T308 and S473) and activation of Akt, a serine/ threonine (S/T) kinase. A link between Akt and GLUT4 translocation was revealed by Gustav Lienhard's group [11, 14], which discovered that insulin-mediated TBC1D4 phosphorylation is important for GLUT4 translocation in 3T3-L1 adipocytes. TBC1D4 includes a Rab-GAP domain, two phosphotyrosine-binding (PTB) domains, a calmodulinbinding domain (CBD) and five canonical Akt phosphomotifs (Fig. 1).

Sano et al [14] reported that insulin-stimulated GLUT4 translocation in 3T3-L1 adipocytes was diminished by overexpression of TBC1D4 with a mutation (serine or threonine to alanine) preventing phosphorylation of either S588 or T642
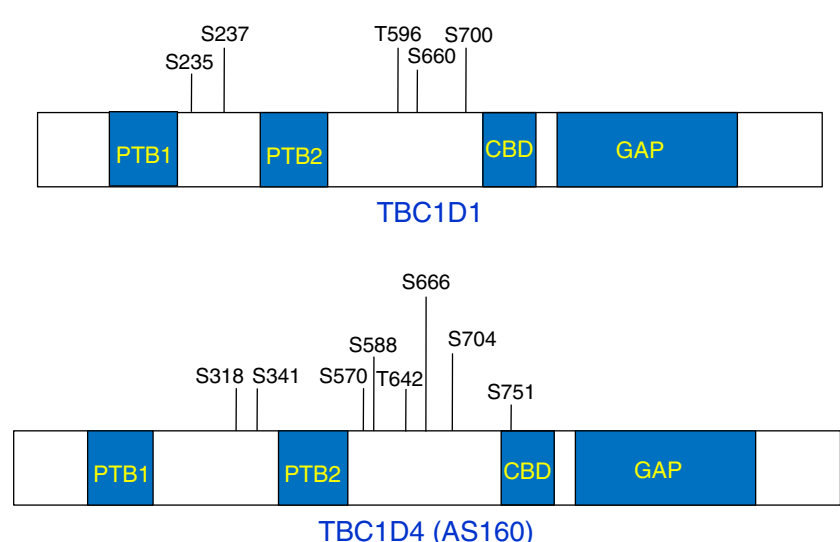

Fig. 1 Schematic diagrams illustrating some of the prominent structural features of TBC1D1 and TBC1D4 (Akt substrate of $160 \mathrm{kDa}, \mathrm{AS160}$ ). Each protein includes a Rab-GAP domain, two PTB domains and a CBD. The potential phosphomotifs that are depicted for each protein are designated by the amino acid numbers for the respective human proteins, and each of these sites can be phosphorylated in response to insulin and/or exercise in skeletal muscle 
(with the T642A mutation having a greater inhibitory effect than the S588A mutation), and simultaneous S588A/T642A mutations had a greater inhibitory effect than either single mutation. Simultaneous mutation to prevent phosphorylation on six phosphomotifs (S318A, S341A, S588A, T642A, S570A and S751A) caused little further inhibition. Analysis of adipocytes expressing a mutation that eliminated Rab-GAP activity (R973K mutant), independently or together with four Akt phosphomotif mutations (S318A, S588A, T642A, S751A; 4P mutant), revealed that site-selective phosphorylation by TBC1D4 is essential for insulin-stimulated GLUT4 translocation only if there is an active Rab-GAP domain. They proposed that insulin-stimulated phosphorylation of TBC1D4 by Akt on selected phosphomotifs leads to the attenuation of TBC1D4's activation of Rab-GTPase proteins associated with GLUT4 vesicles, favouring Rab-GTP accumulation and GLUT4 vesicle exocytosis. It was subsequently demonstrated that 14-3-3 proteins bind with high affinity to phosphorylated T642 and with low affinity to phosphorylated S341 [21], and 14-3-3 binding to TBC1D4 may be important for insulinstimulated GLUT4 translocation [22, 23].

There are more than 60 predicted members of the Rab-GTPases in the human genome. In a cell-free assay, The GAP domain of TBC1D4 showed activity against Rab2A, -8A, -10 and -14 [24]. In 3T3-L1 adipocytes, Rab10 was crucial for insulin-stimulated GLUT4 translocation [25], but in L6 myocytes, Rab8A and Rab13 (but not Rab10) were implicated as TBC1D4 targets required for insulin-stimulated GLUT4 translocation [26]. There are no known specific Rab-GTPases targeted by TBC1D4 in human myocytes or muscle tissue from any species.

There is controversy regarding which of the specific aspects of GLUT4 vesicle traffic are influenced by TBC1D4 [27-29]. One model proposes that TBC1D4 modulates GLUT4 vesicle release from retention without altering vesicle tethering, docking or fusion with cell surface membranes [30]. Another model proposes that after releasing GLUT4 vesicles from retention secondary to TBC1D4 phosphorylation and 14-3-3 binding, the N-terminal PTB domain of TBC1D4 facilitates GLUT4 vesicle docking/fusion with the plasma membrane [31]. Both models are based on the findings of experiments on adipocytes. Neither model has been assessed in muscle.

\section{TBC1D4: insulin-stimulated glucose transport in muscle}

Overexpression of wild-type (WT) TBC1D4 did not alter insulin-stimulated GLUT4 translocation in L6 myocytes, but expressing the 4P-TBC1D4 phosphomutant substantially reduced insulin-stimulated GLUT4 translocation [32, 33]. Expressing 4P-TBC1D4 in L6 myocytes inhibited insulinstimulated GLUT4 translocation, and expression of TBC1D4 with both $\mathrm{R} 973 \mathrm{~K}$ and $4 \mathrm{P}$ mutations relieved this inhibition, demonstrating that an active GAP domain is essential for inhibition of insulin-stimulated GLUT4 translocation by TBC1D4 in myocytes. Insulin-stimulated mouse TA overexpressing 4P-TBC1D4 had $\sim 50 \%$ lower in vivo glucose uptake vs controls [34]. Mice with a T649A-TBC1D4 knockin mutation (preventing phosphorylation at mouse T649-TBC1D4, equivalent to human T642-TBC1D4) had $\sim 20 \%$ lower glucose uptake by the TA and quadriceps during a euglycaemichyperinsulinaemic clamp vs controls [35]. Furthermore, insulin-stimulated glucose uptake by and cell surface GLUT4 content of isolated soleus were lower for T649A-TBC1D4 knockin vs WT mice. Results for Akt2-null mice revealed that $\mathrm{Akt} 2$ is essential for increased glucose uptake by muscles in the presence of physiological insulin concentrations [36, 37], and Akt2 is crucial for muscle insulin-stimulated pT642TBC1D4 [38]. Compelling evidence links Akt2-dependent pT642-TBC1D4 with insulin-regulated glucose uptake in muscle. Based on 3T3-L1 adipocyte results [14], it can reasonably be predicted that S588-TBC1D4 may regulate muscle glucose uptake, but a point mutation of S588-TBC1D4 has not been studied in muscle.

\section{TBC1D1: insulin-stimulated glucose transport by muscle}

TBC1D1 and TBC1D4 share 50\% identity, and both include a GAP domain (79\% identity), two PTB domains and a CBD (Fig. 1). TBC1D1 and TBC1D4 are highly conserved, with the mouse protein sharing $\sim 90 \%$ identity with the respective human protein [16]. TBC1D4 has multiple canonical Akt phosphorylation motifs, whereas TBC1D1 only has one (T596-TBC1D1, which corresponds to T642-TBC1D4) [39].

Insulin-stimulated glucose transport has been studied in adipocytes with TBC1D1 overexpression, but because endogenous TBC1D1 expression is very low in white adipose tissue, using muscle to study the function of TBC1D1 is more straight-forward. Insulin-stimulation increases pT596TBC1D1 in human [40] and mouse [41, 42] muscles. Insulin increases pT596-TBC1D1, but not pS237-TBC1D1 in L6 myocytes [17]. In $\mathrm{C} 2 \mathrm{C} 12$ myocytes, insulin increased phosphorylation of multiple TBC1D1-phosphosites (including S235, T505, S507, S527 and S627) identified by mass spectrometry [43]. Although pS237-TBC1D1 and pT596TBC1D1 are 14-3-3 binding sites, insulin did not increase the ability of TBC1D1 to bind 14-3-3 [17]. Insulin increased pS235-TBC1D1 and binding of the adaptor protein containing PH domain, PTB domain and leucine zipper motif 2 (APPL2) to TBC1D1 in myocytes and mouse EDL [44]. In myocytes overexpressing APPL2, the binding of TBC1D1 to APPL2 required $\mathrm{pS235-TBC1D1}$ and attenuated insulin-stimulated pT596-TBC1D1 and glucose uptake [44]. Cells expressing a T596D-TBC1D1 phospho-mimic mutant were protected from 
APPL2 overexpression-induced insulin resistance. Experiments with Akt2-null mice revealed that Akt2 is essential for the insulin-mediated increase of pT596-TBC1D1 in TA [42]. S237-TBC1D1 did not become phosphorylated in insulinstimulated human [40], mouse [41] or rat [45] muscle. In vivo insulin-stimulated glucose uptake by mouse TA was unaltered by overexpression of WT TBC1D1 or mutated TBC1D1 (S237A, T505A, T596A and S627A) [46]. Unlike TBC1D4, the influence of TBC1D1 on specific steps of insulinstimulated GLUT4 trafficking has not been reported. The creation of mice with a T596A-TBC1D1 knockin mutation would help clarify the role of site-specific phosphorylation of TBC1D1 in muscle insulin-stimulated glucose transport.

\section{TBC1D4: exercise-stimulated glucose transport in muscle}

Studying contractile activity by ex vivo muscle enables the direct effects of contraction to be studied in isolation (from systemic consequences of exercise, such as blood-flow, hormones, etc.), as well as precise tension measurement and exact hormone or drug doses. Contractile activity by isolated rat epitrochlearis led to greater PAS-TBC1D4 [12] and pT642TBC1D4 [45]. However, it is also crucial to understand the more complex effects of in vivo exercise. PAS-TBC1D4 [47], pT642-TBC1D4 and pS588-TBC1D4 [45, 48-50] were increased in rat epitrochlearis both immediately and 3-4 $\mathrm{h}$ after exercise. Endurance exercise also increases TBC1D4 phosphorylation in human muscle. Cycling (67\% peak oxygen consumption $\left.\left[\dot{V}_{2 \text { peak }}\right]\right)$ caused increased PAS-TBC1D4 at 60 and $90 \mathrm{~min}$, but not at 1,10 or $30 \mathrm{~min}$ [51]. PAS-TBC1D4 increased immediately and $3 \mathrm{~h}$ after exercise (60 min cycling, $\left.60 \% \dot{V} \mathrm{O}_{2 \text { peak }}\right)$, whereas the 14-3-3 binding capacity of TBC1D4 increased only immediately post exercise [52]. Cycling (20 min, 77\% $\dot{V} \mathrm{O}_{2 \text { peak }}$ ) increased pS711-TBC1D4 [53], but PAS-TBC1D4 was lower after the same protocol [51]. One-legged exercise (60 min, $80 \%$ peak work rate) caused increased TBC1D4 phosphorylation on S341, S588, T642, S704, and S751, but not on S318 or S666 [54]. Cycling (30 min, 70\% maximal oxygen consumption) induced greater pS711-TBC1D4 in obese individuals [55]. In summary, endurance exercise can elevate TBC1D4 phosphorylation on multiple sites in rat and human muscles.

TBC1D4 phosphorylation has also been assessed in human muscle after strength-type or sprint exercise. PAS-TBC1D4 was unaltered [56] or reduced immediately after strength-type exercise [57], but elevated at 1, 2 [58] or $24 \mathrm{~h}$ [56] exercise. PAS-TBC1D4 was unaltered immediately after sprint cycling (30 or $120 \mathrm{~s}$ ) [51]. Furthermore, sprint cycling (30 s) did not elevate pT642-TBC1D4 immediately, or at $30 \mathrm{~min}$ or $4 \mathrm{~h}$ post exercise, but values were increased at $2 \mathrm{~h}$ post exercise [59]. Immediately after longer duration interval cycling $(4 \times 4 \mathrm{~min})$,
pT642-TBC1D4 exceeded resting values for obese, nondiabetic men [60]. The mechanisms and consequences of increased TBC1D4 phosphorylation often observed after sprint or strength-type exercise are uncertain.

TBC1D4 phosphorylation has also been studied in mice after contractions (in situ or ex vivo) or in vivo exercise. Mice performing treadmill exercise (30 $\mathrm{min}$ ) had greater gastrocnemius levels of pT642-TBC1D4 [38]. In contrast, $60 \mathrm{~min}$ of treadmill exercise did not alter TBC1D4 phosphorylation in mouse EDL at S588, T642, S704 or S751, and caused decreased EDL pS341-TBC1D4 [54]. In the soleus, there were no exercise effects on pS588-TBC1D4 or pS704-TBC1D4 concomitant with reduced pS341-TBC1D4, pT642-TBC1D4 and pS751-TBC1D4 [54]. Ex vivo contraction of mouse soleus caused greater pS704-TBC1D4 [54], unaltered pS588-TBC1D4 and reduced pT642-TBC1D4 and pS751TBC1D4 [54]. Mass spectrometric analysis of mouse gastrocnemius immediately after in situ contractions revealed greater TBC1D4 phosphorylation (S269, S588, S591, S609, T642 and S781) [53]. In situ contraction by mouse gastrocnemius or TA elevated pS588-TBC1D4, reduced pT642-TBC1D4 and did not alter S318, S341 or S751 phosphorylation [61]. Disparate results in mice vs humans and rats may reflect species differences and/or other experimental differences (e.g. muscles studied, exercise/contraction protocols).

In contrast to the strong evidence linking TBC1D4 phosphorylation to insulin-stimulated glucose transport, the evidence is less convincing for the role of TBC1D4 role in contraction/exercise-stimulated glucose transport. In isolated rat epitrochlearis, a phosphatidylinositol 3-kinase (PI3K) inhibitor (wortmannin) prevented contraction-stimulated PAS-TBC1D4 without attenuating contraction-stimulated glucose transport, and an AMPK-inhibitor (compound C) reduced contraction-stimulated glucose transport without altering PAS-TBC1D4 [12, 62]. Direct evidence against an essential role for pT642-TBC1D4 is provided by the finding that contraction-stimulated glucose uptake in mouse EDL (in situ or ex vivo contractions) was not different for T649A knockin vs WT mice [61]. The absence of contractionstimulated pT649-TBC1D4 in gastrocnemius and TA from WT mice also argues against pT649-TBC1D4 being required for contraction-stimulated glucose uptake. T649A knockin mice are insulin resistant [35], suggesting that pT649-TBC1D4 is more important for insulin-stimulated vs contraction-stimulated glucose uptake. Electroporation of mouse TA with WT TBC1D4 reduced contraction-stimulated glucose uptake, and there was a further $\sim 20-25 \%$ deficit of contraction-stimulated glucose uptake for muscles transfected with the 4P-TBC1D4 mutant, suggesting the TBC1D4 phosphorylation may influence contraction-stimulated glucose uptake $[34,63,64]$. The explanation for the differing results of the 4P-TBC1D4 model vs the T649A knockin model on contraction-stimulated glucose uptake is uncertain, but may relate to the six- to eightfold 
overexpression of TBC1D4 in the 4P-TBC1D4 model, and/or the mutations of three additional phosphomotifs (including S588) in 4P-TBC1D4. Unexpectedly, muscles electroporated with R/K-TBC1D4 or R/K-4P-TBC1D4 mutants had greater contraction-stimulated glucose uptake vs empty vector controls. It is unclear why overexpression of WT TBC1D4, $\mathrm{R} / \mathrm{K}-\mathrm{TBC} 1 \mathrm{D} 4$ and $\mathrm{R} / \mathrm{K}-4 \mathrm{P}-\mathrm{TBC} 1 \mathrm{D} 4$ influenced contractionstimulated glucose uptake, but these constructs did not alter insulin-stimulated glucose uptake in mouse TA [34]. Perhaps contraction/exercise and insulin regulate different subcellular pools of TBC1D4.

In human muscle, exercise can increase phosphorylation of S704-TBC1D4 (a 5' AMP-activated kinase [AMPK] consensus phosphomotif) [53]. Contraction-stimulated glucose uptake was not different in mouse TA that was electroporated with WT TBC1D4 vs muscle electroporated with TBC1D4 mutated to prevent phosphorylation at this site [53]. These results argue against S704 being essential for contraction-stimulated glucose uptake.

Even if TBC1D4 phosphorylation is not a major regulator of contraction-stimulated glucose uptake, it is important to determine which $\mathrm{S} / \mathrm{T}$ kinase(s) participate in exercise/ contraction-induced changes in TBC1D4 phosphorylation because TBC1D4 phosphorylation may influence other cellular functions. Geraghty et al [21] evaluated the ability of purified $\mathrm{S} / \mathrm{T}$ kinases (Akt, AMPK, serum- and glucocorticoidresponsive kinase 1 [SGK1] and $90 \mathrm{kDa}$ ribosomal S6 kinase 1 [RSK1]) to phosphorylate TBC1D4 in a cell-free assay. Each of these S/T kinases strongly increased pS588-TBC1D4, and all but AMPK strongly increased pT642-TBC1D4 (AMPK weakly increased pT642-TBC1D4). The binding of TBC1D4 to 14-3-3 in an overlay assay was strongly increased by purified Akt, SGK1 or RSK1, and was increased to a much smaller extent by AMPK. In cell-free assays, recombinant AMPK (but not Akt1, Akt2 or protein kinase $\mathrm{C} \zeta$ ) increased pS704-TBC1D4 [53], and recombinant $\mathrm{Ca}^{2+} /$ calmodulin-dependent protein kinase kinase $\alpha(\mathrm{CaMKK} \alpha)$ increased pS588TBC1D4, but not pT642-TBC1D4 [65]. Cell-free experiments are informative, but additional approaches are essential to elucidate the physiological regulation of TBC1D4 phosphorylation.

Of the five S/T kinases known to increase TBC1D4 phosphorylation in cell-free assays, AMPK has been the most frequently studied to elucidate exercise/contraction effects. The AMPK-activator 5-aminoimidazole-4-carboxamide-1- $\beta$ D-ribofuranoside (AICAR) induced greater PAS-TBC1D4 in rat epitrochlearis, suggesting that AMPK phosphorylated TBC1D4 [12]. Many studies have demonstrated that muscle AMPK is activated immediately after endurance exercise or ex vivo contractions [2-4]. Immediately after $60 \mathrm{~min}$ of one-legged exercise by humans, TBC1D4 phosphorylation (S341, S588, T642 and S704) was increased, but only pS341-TBC1D4 and pS704-TBC1D4 were significantly correlated with $\alpha 2 / \beta 2 / \gamma 1$ AMPK activity [54]. Increased pT642-TBC1D4 in the TA after in situ contractions was blunted in transgenic mice with muscle-specific expression of inactive AMPK $\alpha 2$ vs WT mice [66].

Some [12, 67-69], but not all [70-72], studies have demonstrated that Akt is activated immediately after electrically stimulated contractions. Increased Akt activation has been observed in muscles immediately after in vivo endurance exercise without added insulin in some [68, 73], but not all [74-76], studies. There was no attenuation of pT642-TBC1D4 in EDL from Akt2-null mice after in situ contractions [38].

Little is known about the possible roles of SGK1, RSK1 or CaMKK $\alpha$ in exercise-stimulated TBC1D4 phosphorylation. Endurance exercise induced greater pT642-TBC1D4, pS588TBC1D1 and pT173-AMPK in rat epitrochlearis without increased phosphorylation of regulatory sites on Akt, SGK1 or RSK1 [48].

Increased cytosolic calcium is essential for muscle contraction and a potential trigger for contraction-stimulated glucose transport. Although overexpression of TBC1D4 with a CBD mutation preventing TBC1D4 binding to calmodulin did not alter insulin-stimulated glucose uptake, this mutation modestly reduced contraction-stimulated glucose uptake by mouse TA [64]. Overexpressing TBC1D4 with simultaneous CBD and $4 \mathrm{P}$ mutations in mouse TA did not cause a greater reduction in contraction-stimulated glucose uptake than with either mutation alone [64].

\section{TBC1D1: exercise/contraction-stimulated glucose transport in muscle}

Many studies have evaluated the effect of contractions on TBC1D1 phosphorylation in rodents. Contraction increased PAS-TBC1D1 in mouse TA [18] and rat epitrochlearis [62]. In isolated rat epitrochlearis, contraction-stimulated PAS-TBC1D1 was eliminated by the AMPK-inhibitor compound $C$ with attenuation of $\mathrm{pT}$ 172-AMPK and glucose transport [62]. Contraction elevated pS237-TBC1D1 (an AMPK consensus motif) in rat epitrochlearis [45] and increased pS237-TBC1D1 and pT596-TBC1D1 in the EDL of WT mice, but phosphorylation was not increased on either site in AMPK $\alpha 2$ kinase-dead mice [41]. Contraction-stimulated EDL glucose uptake in AMPK $\alpha 2$ kinase-dead mice was lower than that in WT mice [77]. Contraction increased the binding capacity of TBC1D1 for 14-3-3 in EDL in WT mice, but not in AMPK $\alpha 2$ kinase-dead mice [41]. Contraction by WT mouse TA increased pS237-TBC1D1 [42, 61] and pS666-TBC1D1, but did not alter pT596-TBC1D1 [42]. Contraction effects on these sites were markedly lower for AMPK $\alpha 2$ inactive transgenic vs WT mice [42], whereas contraction effects were similar for Akt2-null vs WT mice 
[41]. In isolated EDL from WT mice, contraction induced increases in pS237-TBC1D1, pT596-TBC1D1, pS660TBC1D1 and pS700-TBC1D1, and these effects were either eliminated or almost eliminated in AMPK $\alpha 2$-null mice [54]. Contraction-stimulated glucose uptake in the EDL was unaffected for AMPK $\alpha 1$-null mice vs WT mice [78]. However, AMPK $\alpha 2$-null mice have a compensatory increase in AMPK $\alpha 1$ expression. Muscle-specific $\beta 1 \beta 2$-null mice have undetectable muscle AMPK activity accompanied by markedly reduced contraction-stimulated (isolated EDL and soleus) and in vivo treadmill exercise-stimulated (gastrocnemius and soleus) glucose uptake [79]. Contraction-stimulated PAS-TBC1D1 was also nearly eliminated in isolated EDL of $\beta 1 \beta 2$-null mice. Treadmill exercise (60 min) increased p237-TBC1D1, pT596TBC1D1, pS660-TBC1D1 and pS700-TBC1D1 in the EDL, but not the soleus, and pS596-TBC1D1 was unaltered by exercise in EDL or soleus [54]. This treadmill protocol induced an increase in pT172-AMPK in the EDL, but not the soleus, and exercise decreased pS473-Akt and pT308-Akt in both muscles. In vivo contraction-stimulated glucose uptake was $\sim 30-45 \%$ lower in mouse TA overexpressing TBC1D1 with a mutation preventing phosphorylation on four phosphomotifs (including S237) [42, 46]. Contraction or exercise usually elevates pS237-TBC1D1 of rodent muscle, and this increase appears to be secondary to greater AMPK activity and linked to greater contraction/exercise-stimulated glucose uptake.

Several studies have assessed the effect of exercise on TBC1D1 phosphorylation in human muscle. Increased pS237-TBC1D1, but not pT596-TBC1D1, was observed immediately after high-intensity ( $30 \mathrm{~s}$ or $2 \mathrm{~min}$ ) or moderateintensity (20 min, $77 \% \dot{V} \mathrm{O}_{2 \text { peak }}$ ) cycling [80]. These protocols also increased the binding capacity of TBC1D1 for 14-3-3. Immediately after cycling (30 $\left.\mathrm{min}, 70 \% \dot{V} \mathrm{O}_{2 \text { peak }}\right), \mathrm{pS} 237$ TBC1D1 was increased, but pS596-TBC1D1 was not, in muscles of obese people [55]. Immediately after one-legged exercise (60 min), there were increases in pS237-TBC1D1 and pT596TBC1D1 that correlated with exercise-induced increases in $\alpha 2 / \beta 2 / \gamma 3$ AMPK activity [54]. Exercise also produced elevations in pS660-TBC1D1 and pS700-TBC1D1, but not in the binding capacity of TBC1D1 for 14-3-3. S237-TBC1D1 and T596-TBC1D1 phosphorylation were increased at $30 \mathrm{~min}$ after cycling (60 min, $65 \% \dot{V} \mathrm{O}_{2 \text { peak }}$ ) [81]. As in rodents, human exercise consistently increases pS237-TBC1D1, but does not always increase pT596-TBC1D1. Increased S237-TBC1D1 phosphorylation is a promising candidate in the regulation of exercise-dependent glucose uptake by human muscle.

\section{TBC1D1 and TBC1D4: insulin sensitivity after acute exercise}

Several hours after one exercise session by healthy individuals, insulin-stimulated glucose transport is increased secondary to elevated GLUT4 translocation without increased GLUT4 expression or enhanced proximal insulin signalling, including insulin receptor binding, IRS1 tyrosine phosphorylation, IRS1-associated PI3K activity and Akt activity $[8,49$, $50,75,82-85]$. Despite unaltered proximal signalling, acute exercise can increase TBC1D4 phosphorylation and glucose uptake in muscle stimulated with a physiological insulin dose (Fig. 2) [40, 45, 47-50, 86]. Isolated rat epitrochlearis incubated with a physiological insulin concentration at $3-4 \mathrm{~h}$ post exercise had increased PAS-TBC1D4 [47], pS588-TBC1D4 $[48,50]$ and pT642-TBC1D4 [45, 48-50]. Increased insulinstimulated glucose transport tracked closely with changes in pT642-TBC1D4: both persisted for $27 \mathrm{~h}$ post exercise in muscles from rats that were not fed chow after exercise, but consuming chow for $3 \mathrm{~h}$ post exercise reversed these exercise effects [49]. Wojtaszewski and colleagues probed the relationship between TBC1D4 phosphorylation and insulinstimulated glucose uptake in healthy humans after onelegged exercise for $60 \mathrm{~min}[40,86]$. Muscle glucose uptake was measured for the non-exercising and exercising leg during a clamp (with a physiological insulin concentration). Pehmøller et al [40] reported that insulin-stimulated muscle from the exercised vs non-exercised leg had greater TBC1D4 phosphorylation on S318, S341, S588, T642, S704 and S751, whereas Treebak et al [86] reported increased TBC1D4
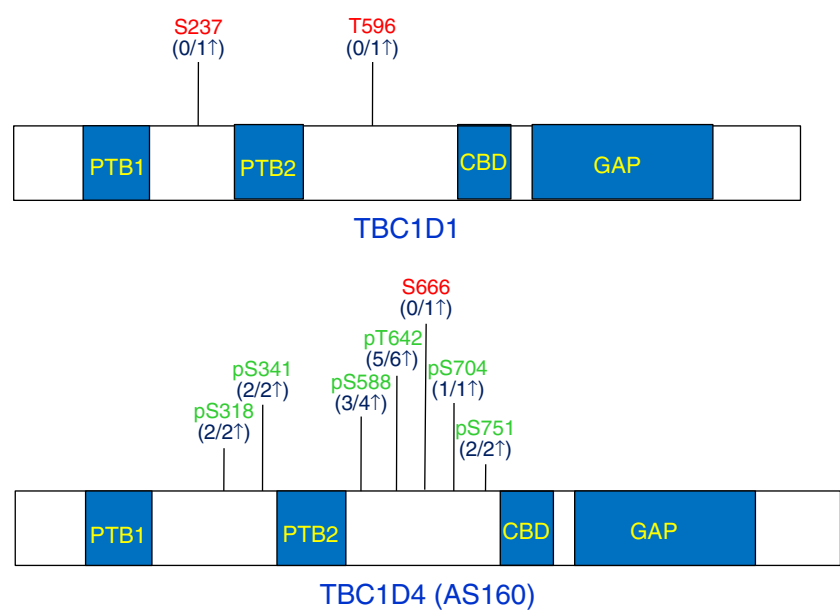

Fig. 2 Effect of acute exercise on subsequent site-specific TBC1D1 and TBC1D4 phosphorylation in normal muscle in response to a physiological insulin dose. This figure depicts the results of published experiments for acute endurance exercise that resulted in a subsequent elevation of insulin-stimulated glucose uptake with a physiological insulin dose in non-insulin resistant, non-diabetic rats or humans [40, 45, 47-50, 86]. The phosphomotifs shown in red font did not have an exercise-induced increase in insulin-stimulated phosphorylation compared with unexercised control muscle. The phosphomotifs shown in green font were found in most of the published studies to have an exercise-induced increase in insulin-stimulated phosphorylation vs unexercised controls. The numbers in parentheses below each phosphomotif represent the ratio of the number of publications with a significant exercise-induced increase in insulinstimulated phosphorylation and the total number of publications making the relevant exercise comparison 
phosphorylation on S318, S341 and S751, with a nonsignificant trend for greater pS588-TBC1D4, and unaltered pT642-TBC1D4 and pS666-TBC1D4. The binding affinity of TBC1D4 for 14-3-3 (measured by overlay assay) was unaltered by exercise in both studies. High-intensity interval cycling $\left(4 \times 4 \mathrm{~min} ; 90-95 \%\right.$ heart-rate $\left._{\text {peak }}\right)$ induced greater glucose infusion during a euglycaemic-hyperinsulinaemic clamp with a physiological insulin dose, but did not significantly increase muscle pT642-TBC1D4 determined $\sim 3 \mathrm{~h}$ post exercise [60]. In isolated mouse plantaris incubated with a supraphysiological insulin dose $24 \mathrm{~h}$ after treadmill exercise (90 min duration), there was increased insulin-stimulated glucose uptake with no exercise-effect on pT642-TBC1D4 [87]. Most, but not all, studies in healthy rats or humans using a physiological insulin dose after acute exercise have reported increased TBC1D4 phosphorylation.

Prior exercise does not elevate the phosphorylation of all Akt substrates in insulin-stimulated muscles. There was no increase in PAS-TBC1D1 in insulin-stimulated rat epitrochlearis at 3 or $27 \mathrm{~h}$ post exercise [49]. Neither pT596TBC1D1 nor pS237-TBC1D1 differed for exercised vs nonexercised insulin-stimulated muscles sampled at $\sim 5-7 \mathrm{~h}$ post exercise [40]. There were also no exercise effects on insulinstimulated pS21/9-glycogen synthase kinase 3 (GSK3) in rat epitrochlearis at 3-4 h post exercise [47], or on insulinstimulated pS21-GSK3 in human muscle at $\sim 4-6 \mathrm{~h}$ post exercise [83]. The effect of exercise on pTBC1D4 is at least somewhat specific, although it will be important to evaluate additional Akt substrates. Apparent uncoupling between the extent of Akt activation and the levels of phosphorylation of various substrates (and even individual phosphosites on one substrate) is not unique to the post-exercise condition [85-87].

What are the mechanisms that account for greater TBC1D4 phosphorylation in insulin-stimulated muscle several hours post exercise without greater Akt activity? Perhaps prior exercise leads to greater activation of other $\mathrm{S} / \mathrm{T}$ kinases that can phosphorylate TBC1D4. However, insulin did not increase the phosphorylation of activation sites for AMPK, SGK1 or RSK1 in epitrochlearis from unexercised or exercised rats [48]. Protein phosphorylation represents the balance between kinases and phosphatases. It is uncertain which $\mathrm{S} / \mathrm{T}$ protein phosphatase(s) dephosphorylates TBC1D4, but protein phosphatase 2A (PP2A) is abundantly expressed by muscle. However, exercise did not alter Y307 phosphorylation or L309 methylation of the catalytic subunit of PP2A [48].

Each of the direct determinants of protein phosphorylation (i.e. relevant kinase(s) and phosphatases and the substrate itself) might be regulated by interactions with other protein binding partners, various post-translational modifications, allosteric modulation and/or subcellular localisation (Fig. 3). Assuming Akt is the relevant kinase, conventional cell-free Akt enzyme assays do not address allosteric modulation or subcellular localisation, and immunoprecipitated Akt may not retain all endogenous protein binding partners or all posttranslational modifications. The first step in understanding the role of $\mathrm{S} / \mathrm{T}$ protein phosphatase(s) would be to identify the relevant enzymes that dephosphorylate TBC1D4. Several proteins have been identified as TBC1D4 binding partners, including 14-3-3, RuvB-like 2 (RUVBL2), receptorinteracting protein of $140 \mathrm{kDa}$ (RIP140) and cytoplasmic linker protein-protein $59 \mathrm{kDa}$ protein (ClipR-59) [22, 88-91]. The effect of exercise on the capacity of TBC1D4 binding to 14-3-3 using an overlay assay has been reported $[40,86]$, but the direct binding of endogenous 14-3-3 (or any of these other proteins) to TBC1D4 has not been reported for insulin-stimulated muscle after acute exercise.

Improvements in insulin sensitivity can be observed in insulin-resistant individuals after acute exercise, but few studies have probed the mechanism that underlies these improvements. Castorena et al [50] found that, 3-4 h after endurance exercise, insulin-stimulated glucose uptake was elevated in isolated epitrochlearis from normal rats (eating a low-fat diet, LFD) and insulin-resistant rats eating a high-fat diet (HFD). Insulin-stimulated Akt activity was unaltered in both dietgroups at $3 \mathrm{~h}$ post exercise. In insulin-stimulated muscles from LFD-rats at $3 \mathrm{~h}$ post exercise, the levels of pS588-TBC1D4 and pT642-TBC1D4 exceeded those in diet-matched sedentary controls. At $3 \mathrm{~h}$ post exercise, the insulin-resistant HFD rats had increased pS588-TBC1D4 (but not pT642-TBC1D4) in insulin-stimulated muscles vs diet-matched sedentary controls. After exercise, pS588-TBC1D4 and pT642-TBC1D4 in insulin-stimulated muscles from HFD animals equalled the values in sedentary LFD controls. However, exercise failed to elevate insulin-stimulated glucose uptake, pS588-TBC1D4 or pT642-TBC1D4 in the HFD group to values as great as those in the post-exercise LFD-group. In both diet-groups, insulinstimulated glucose uptake consistently tracked with TBC1D4 phosphorylation post exercise.

Pehmøller et al [40] induced insulin resistance in healthy humans by infusing intralipid for $7 \mathrm{~h}$ and studied the same individuals in a control saline infusion condition. In both trials, participants performed $60 \mathrm{~min}$ of one-legged exercise $\sim 5.5 \mathrm{~h}$ prior to a clamp with a physiological insulin concentration. Exercised vs non-exercised legs had greater glucose uptake and TBC1D4 phosphorylation (S318, S341, S588, T642, S704 and S751) in both trials, without exercise effects on proximal insulin signalling (IRS-1-PI3K or Akt phosphorylation). Similar to the results for insulin-resistant rats [50], exercise improved insulin-stimulated glucose uptake by insulin-resistant muscles levels equivalent to normal, nonexercised values [40]. Also similar to the findings in insulinresistant rats, insulin-stimulated glucose disposal was lower in the post-exercise lipid-infusion trial compared with the postexercise saline-infusion trial.

In HFD-fed streptozotocin-induced diabetic rats, $\sim 8-16 \mathrm{~h}$ post exercise, glucose transport and pS473-Akt were 


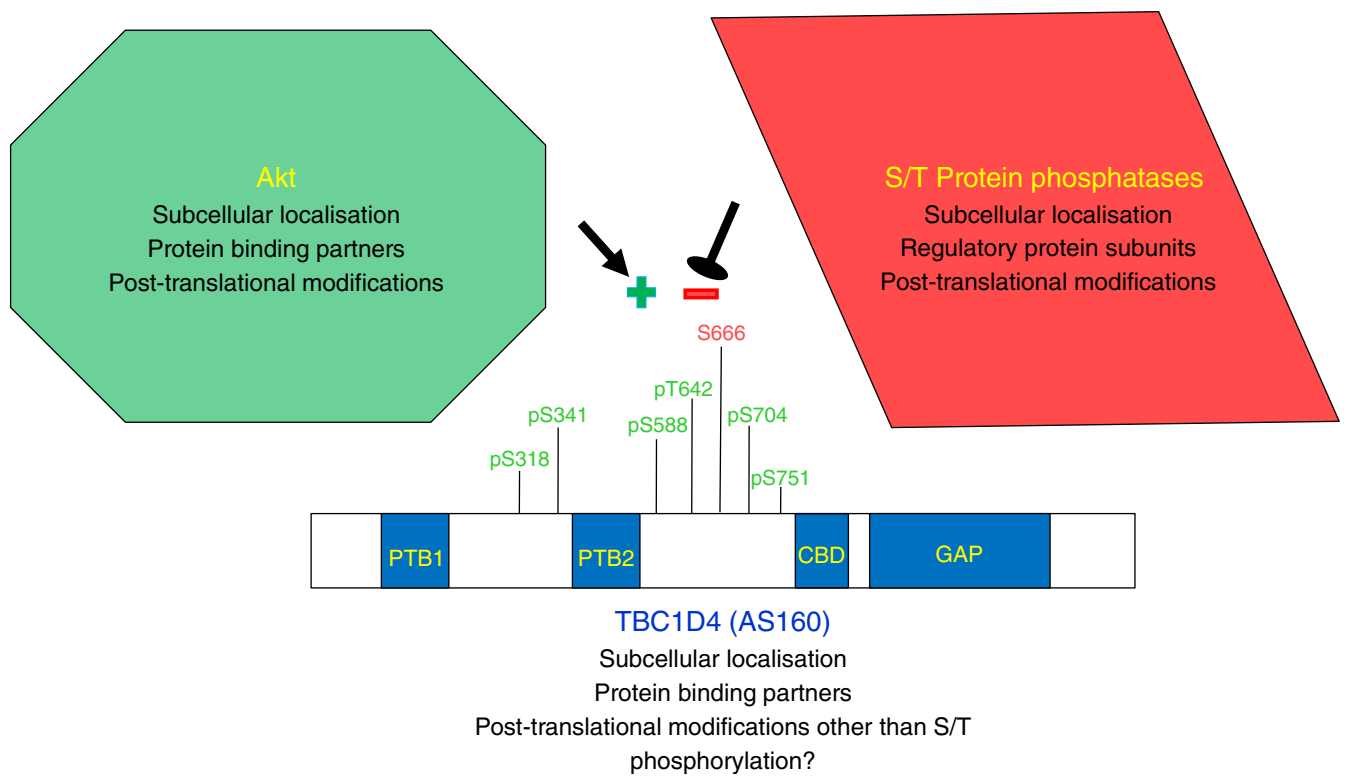

Fig. 3 Potential mechanisms for increased TBC1D4 phosphorylation in the absence of greater Akt activity in insulin-stimulated muscle after acute exercise. This figure depicts potential mechanisms that might account for acute exercise effects on TBC1D4 phosphorylation in insulin-stimulated muscle. One exercise session can increase the subsequent $(\sim 3-27 \mathrm{~h}$ post exercise) TBC1D4 phosphorylation in insulin-stimulated muscle without an increase in Akt activity determined by a conventional Akt enzyme activity assay (using immunoprecipitated Akt with an Akt peptide substrate) $[49,50]$. Muscle TBC1D4 phosphorylation depends on the relevant kinase activity, the relevant $\mathrm{S} / \mathrm{T}$ phosphatase activity and the

increased in isolated soleus with a supraphysiological insulin dose concomitant with a non-significant trend for increased pT642-TBC1D4 [92]. In old (24 months) rats, there was increased insulin-stimulated glucose uptake by isolated epitrochlearis and soleus with two submaximally effective insulin doses at $3 \mathrm{~h}$ post exercise [93]. Exercise increased pT642-TBC1D4 in the epitrochlearis (but not soleus) with only one of the submaximally effective insulin doses studied, and exercise did not significantly increase pS588-TBC1D4 in either muscle, regardless of insulin dose [93]. Acute exercise can cause insulin-resistant muscles to increase their insulinstimulated glucose uptake to values similar to those in normal, non-exercised controls, and greater TBC1D4 phosphorylation may be related to improved insulin sensitivity post exercise.

\section{TBC1D4: chronic exercise effects on insulin sensitivity}

One exercise session can enhance insulin sensitivity, but regularly performed exercise for several days, weeks or months may produce further improvements [10]. Endurance training (10 weeks) increased glucose disposal during a euglycaemichyperinsulinaemic clamp performed $48 \mathrm{~h}$ after the final exercise bout in obese men with or without type 2 diabetes [94]. Training increased TBC1D4 phosphorylation (S318, S341, S588, T642 and S751) in insulin-stimulated muscles from properties of TBC1D4 that influence its susceptibility to the kinase and phosphatase. A conventional cell-free Akt assay cannot account for the in-cell co-localisation of TBC1D4 with Akt and S/T phosphatases. Enzyme activity of Akt and S/T phosphatases can be influenced by their binding to other proteins. TBC1D4 also binds to regulatory proteins that can influence its phosphorylation. Post-translational modifications (other than $\mathrm{S} / \mathrm{T}$ phosphorylation) can regulate the activity of Akt and some $\mathrm{S} / \mathrm{T}$ phosphatases, but it is currently unknown whether TBC1D4 has any posttranslational modifications other than $\mathrm{S} / \mathrm{T}$ phosphorylation

the diabetic group, despite there being no training effects on pS473-Akt or pT308-Akt. In the non-diabetic group, with greater TBC1D4 phosphorylation (S341, T642 and S751), pS473-Akt was increased but pT308-Akt was not. Endurance training (12 weeks) by young ( 24 years) or old ( 69 years) nondiabetic humans resulted in increased glucose disposal during a clamp ( $\sim 40 \mathrm{~h}$ after final training session) in both age groups [88]. The older group was modestly insulin-resistant compared with the young group. Endurance training increased pS588-TBC1D4 in both groups, increased pT642-TBC1D4 only in the older group, and did not alter pS666-TBC1D4 in either group. Both age groups were also studied following strength training (12 weeks), and glucose disposal during a clamp ( $\sim 40 \mathrm{~h}$ after final training session) increased in both age groups. Strength training increased pT642-TBC1D4 in both age groups, increased pS666-TBC1D4 in the older group only and did not alter pS588-TBC1D4 regardless of age. In both insulin-resistant, non-diabetic HFD-fed rats and streptozotocin-induced diabetic HFD-fed rats, endurance training ( 8 weeks) increased glucose transport by isolated soleus incubated with a supraphysiological insulin dose ( 24-36 h after final training bout) [92]. In both groups, training increased pT642-TBC1D4 in muscles incubated with supraphysiological insulin [92]. Endurance training (4 weeks) increased glucose uptake without increasing pT642-TBC1D4 in perfused hindlimb muscles of rats made insulin resistant by 
a cafeteria diet (24 $\mathrm{h}$ after final training session) [95]. To the best of my knowledge, there are no published reports of training effects on insulin-stimulated TBC1D1 phosphorylation. In some experimental conditions, exercise training induces greater site-specific phosphorylation of TBC1D4 in insulin-stimulated muscle, which, together with other exercise training effects (e.g. increased GLUT4 protein expression [4]) may contribute to improved insulin sensitivity.

\section{TBC1D1-null and TBC1D4-null mice}

Mice with a naturally occurring loss-of-function mutation of the Tbcld1 gene had reduced GLUT4 abundance and ex vivo insulin-stimulated glucose uptake in EDL (high TBC1D1 expression in WT mice), but not in soleus (low TBC1D1 expression in WT mice) [96, 97]. Soleus, gastrocnemius and diaphragm glucose uptake during a euglycaemichyperinsulinaemic clamp was unaltered for TBC1D1deficient mice, but, paradoxically, EDL and TA glucose uptake were greater for TBC1D1-deficient mice [97]. Conventional TBC1D1-null mice had reduced GLUT4 abundance and insulin-stimulated glucose uptake by isolated EDL [98]. TBC1D4 abundance (soleus and EDL), glucose tolerance and insulin tolerance were unaltered in TBC1D1-null mice.

Whole body TBC1D4-null mice had lower GLUT4 in muscles with high TBC1D4 expression in WT mice (soleus, gastrocnemius and vastus lateralis), but not in muscles with low TBC1D4 expression in WT mice (EDL and TA) [99, 100]. Glucose uptake for TBC1D4-null mice was lower for isolated soleus, but not EDL [100]. Soleus Glut4 mRNA was not lower in TBC1D4-null vs WT mice. Muscle TBC1D1 abundance was similar between genotypes. TBC1D4-null mice had reduced soleus and vastus lateralis glucose uptake during a euglycaemic-hyperinsulinaemic clamp [100]. Both TBC1D1 and TBC1D4 deficiency are characterised by muscle-specific insulin resistance that appears to be related to lower GLUT4 abundance. Perhaps in the absence of TBC1D1 or TBC1D4, GLUT4 trafficking rates increase, leading to accelerated GLUT4 protein degradation and lower GLUT4 abundance. It is important to note that musclespecific knockouts of TBC1D1 and TBC1D4 have not yet been evaluated.

\section{Summary of current knowledge and areas for future study}

TBC1D1 and TBC1D4 have distinctive roles in regulating muscle glucose transport. Akt2-dependent pT642-TBC1D4 is required for the full effect of insulin on glucose transport in muscle. However, the functional significance of insulinstimulated increases in the phosphorylation of several other individual TBC1D4 sites (e.g. S588) has not been determined in muscle. Exercise/contraction-induced glucose uptake often coincides with greater TBC1D1 phosphorylation, but there is no information linking specific GLUT4 vesicle trafficking events (retention, tethering, docking, fusion) to TBC1D1-dependent, site-specific phosphorylation in cultured cells or exercised muscle. Greater phosphorylation of TBC1D4, but not TBC1D1, often tracks closely with the enhanced glucose transport in muscle stimulated with a physiological insulin dose after acute exercise. However, the mechanisms accounting for increased TBC1D4 phosphorylation and its causal importance for improved post-exercise insulin sensitivity remain to be established. Future research should focus on the subcellular localisation of TBC1D1 and TBC1D4 because localised subsets of each protein are likely to perform specific functions. In this context, it is interesting that research using 3T3-L1 adipocytes revealed that a phospholipid-binding region of the N-terminus PTB domain of TBC1D4 regulates the localisation of this protein at the plasma membrane and increases GLUT4 docking/fusion with the plasma membrane. Similar experiments are lacking for muscle. Finally, skeletal muscle highly expresses both TBC1D1 and TBC1D4, and elucidating the complementary and antagonistic interactions between these paralog proteins is needed to fully understand their biological roles, which are likely not limited to the regulation of glucose transport.

Funding Research related to this topic is supported by grants to GDC from the National Institutes of Health (DK71771 and AG10026).

Duality of interest The author declares that there is no duality of interest associated with this manuscript.

Contribution statement GDC was the sole contributor to this paper.

\section{References}

1. Constable SH, Favier RJ, Cartee GD, Young DA, Holloszy JO (1988) Muscle glucose transport: interactions of in vitro contractions, insulin, and exercise. J Appl Physiol 64:2329-2332

2. Cartee GD, Wojtaszewski JF (2007) Role of Akt substrate of $160 \mathrm{kDa}$ in insulin-stimulated and contraction-stimulated glucose transport. Appl Physiol Nutr Metab 32:557-566

3. Cartee GD, Funai K (2009) Exercise and insulin: convergence or divergence at AS160 and TBC1D1? Exerc Sport Sci Rev 37:188195

4. Richter EA, Hargreaves M (2013) Exercise, GLUT4, and skeletal muscle glucose uptake. Physiol Rev 93:993-1017

5. Douen AG, Ramlal T, Rastogi S et al (1990) Exercise induces recruitment of the "insulin-responsive glucose transporter". Evidence for distinct intracellular insulin- and exercise-recruitable transporter pools in skeletal muscle. J Biol Chem 265:13427-13430

6. Cartee GD, Young DA, Sleeper MD, Zierath J, Wallberg-Henriksson H, Holloszy JO (1989) Prolonged increase in insulin-stimulated glucose transport in muscle after exercise. Am J Physiol 256:E494-E499 
7. Richter EA, Garetto LP, Goodman MN, Ruderman NB (1982) Muscle glucose metabolism following exercise in the rat: increased sensitivity to insulin. J Clin Invest 69:785-793

8. Maarbjerg SJ, Sylow L, Richter EA (2011) Current understanding of increased insulin sensitivity after exercise - emerging candidates. Acta Physiol (Oxf) 202:323-335

9. Frøsig C, Richter EA (2009) Improved insulin sensitivity after exercise: focus on insulin signaling. Obesity (Silver Spring) 17(Suppl 3):S15-S20

10. Perseghin G, Price TB, Petersen KF et al (1996) Increased glucose transport-phosphorylation and muscle glycogen synthesis after exercise training in insulin-resistant subjects. N Engl J Med 335:13571362

11. Kane S, Sano H, Liu SC et al (2002) A method to identify serine kinase substrates. Akt phosphorylates a novel adipocyte protein with a Rab GTPase-activating protein (GAP) domain. J Biol Chem 277: 22115-22118

12. Bruss MD, Arias EB, Lienhard GE, Cartee GD (2005) Increased phosphorylation of Akt substrate of $160 \mathrm{kDa}$ (AS160) in rat skeletal muscle in response to insulin or contractile activity. Diabetes 54:4150

13. Arias EB, Kim J, Cartee GD (2004) Prolonged incubation in PUGNAc results in increased protein O-Linked glycosylation and insulin resistance in rat skeletal muscle. Diabetes 53:921-930

14. Sano H, Kane S, Sano E et al (2003) Insulin-stimulated phosphorylation of a Rab GTPase-activating protein regulates GLUT4 translocation. J Biol Chem 278:14599-14602

15. Zhang H, Zha X, Tan Y et al (2002) Phosphoprotein analysis using antibodies broadly reactive against phosphorylated motifs. J Biol Chem 277:39379-39387

16. Roach WG, Chavez JA, Miinea CP, Lienhard GE (2007) Substrate specificity and effect on GLUT4 translocation of the Rab GTPaseactivating protein Tbc1d1. Biochem J 403:353-358

17. Chen S, Murphy J, Toth R, Campbell DG, Morrice NA, Mackintosh C (2008) Complementary regulation of TBC1D1 and AS160 by growth factors, insulin and AMPK activators. Biochem J 409:449-459

18. Taylor EB, An D, Kramer HF et al (2008) Discovery of TBC1D1 as an insulin-, AICAR-, and contraction-stimulated signaling nexus in mouse skeletal muscle. J Biol Chem 283:9787-9796

19. Castorena CM, Mackrell JG, Bogan JS, Kanzaki M, Cartee GD (2011) Clustering of GLUT4, TUG, and RUVBL2 protein levels correlate with myosin heavy chain isoform pattern in skeletal muscles, but AS160 and TBC1D1 levels do not. J Appl Physiol 111: 1106-1117

20. Jensen TE, Leutert R, Rasmussen ST et al (2012) EMG-normalised kinase activation during exercise is higher in human gastrocnemius compared to soleus muscle. PLoS One 7:e31054

21. Geraghty KM, Chen S, Harthill JE et al (2007) Regulation of multisite phosphorylation and 14-3-3 binding of AS160 in response to IGF-1, EGF, PMA and AICAR. Biochem J 407:231-241

22. Chen S, Synowsky S, Tinti M, MacKintosh C (2011) The capture of phosphoproteins by 14-3-3 proteins mediates actions of insulin. Trends Endocrinol Metab 22:429-436

23. Ramm G, Larance M, Guilhaus M, James DE (2006) A role for 143-3 in insulin-stimulated GLUT4 translocation through its interaction with the RabGAP AS160. J Biol Chem 281:29174-29180

24. Miinea CP, Sano H, Kane S et al (2005) AS160, the Akt substrate regulating GLUT4 translocation, has a functional Rab GTPaseactivating protein domain. Biochem J 391:87-93

25. Sano H, Eguez L, Teruel MN et al (2007) Rab10, a target of the AS160 Rab GAP, is required for insulin-stimulated translocation of GLUT4 to the adipocyte plasma membrane. Cell Metab 5:293-303

26. Klip A, Sun Y, Chiu TT, Foley KP (2014) Signal transduction meets vesicle traffic: the software and hardware of GLUT4 translocation. Am J Physiol Cell Physiol 306:C879-C886
27. Sakamoto K, Holman GD (2008) Emerging role for AS160/ TBC1D4 and TBC1D1 in the regulation of GLUT4 traffic. Am J Physiol Endocrinol Metab 295:E29-E37

28. Foley K, Boguslavsky S, Klip A (2011) Endocytosis, recycling, and regulated exocytosis of glucose transporter 4 . Biochemistry 50: 3048-3061

29. Rowland AF, Fazakerley DJ, James DE (2011) Mapping insulin/ GLUT4 circuitry. Traffic 12:672-681

30. Brewer PD, Habtemichael EN, Romenskaia I, Mastick CC, Coster AC (2014) Insulin-regulated Glut4 translocation: membrane protein trafficking with six distinctive steps. J Biol Chem 289:17280-17298

31. Tan SX, Ng Y, Burchfield JG et al (2012) The Rab GTPaseactivating protein TBC1D4/AS160 contains an atypical phosphotyrosine-binding domain that interacts with plasma membrane phospholipids to facilitate GLUT4 trafficking in adipocytes. Mol Cell Biol 32:4946-4959

32. Ishikura S, Bilan PJ, Klip A (2007) Rabs 8A and 14 are targets of the insulin-regulated Rab-GAP AS160 regulating GLUT4 traffic in muscle cells. Biochem Biophys Res Commun 353:1074-1079

33. Thong FS, Bilan PJ, Klip A (2007) The Rab GTPase-activating protein AS160 integrates Akt, protein kinase C, and AMP-activated protein kinase signals regulating GLUT4 traffic. Diabetes 56:414 423

34. Kramer HF, Witczak CA, Taylor EB, Fujii N, Hirshman MF, Goodyear LJ (2006) AS160 regulates insulin- and contractionstimulated glucose uptake in mouse skeletal muscle. J Biol Chem 281:31478-31485

35. Chen S, Wasserman DH, MacKintosh C, Sakamoto K (2011) Mice with AS160/TBC1D4-Thr649Ala knockin mutation are glucose intolerant with reduced insulin sensitivity and altered GLUT4 trafficking. Cell Metab 13:68-79

36. Cho H, Mu J, Kim JK et al (2001) Insulin resistance and a diabetes mellitus-like syndrome in mice lacking the protein kinase Akt2 (PKB beta). Science 292:1728-1731

37. McCurdy CE, Cartee GD (2005) Akt2 is essential for the full effect of calorie restriction on insulin-stimulated glucose uptake in skeletal muscle. Diabetes 54:1349-1356

38. Kramer HF, Witczak CA, Fujii N et al (2006) Distinct signals regulate AS160 phosphorylation in response to insulin, AICAR, and contraction in mouse skeletal muscle. Diabetes 55:2067-2076

39. Chavez JA, Roach WG, Keller SR, Lane WS, Lienhard GE (2008) Inhibition of GLUT4 translocation by Tbc1d1, a Rab GTPaseactivating protein abundant in skeletal muscle, is partially relieved by AMP-activated protein kinase activation. J Biol Chem 283: 9187-9195

40. Pehmøller C, Brandt N, Birk JB et al (2012) Exercise alleviates lipid-induced insulin resistance in human skeletal muscle-signaling interaction at the level of TBC1 domain family member 4 . Diabetes 61:2743-2752

41. Pehmøller C, Treebak JT, Birk JB et al (2009) Genetic disruption of AMPK signaling abolishes both contraction- and insulin-stimulated TBC1D1 phosphorylation and 14-3-3 binding in mouse skeletal muscle. Am J Physiol Endocrinol Metab 297:E665-E675

42. Vichaiwong K, Purohit S, An D et al (2010) Contraction regulates site-specific phosphorylation of TBC1D1 in skeletal muscle. Biochem J 431:311-320

43. Peck GR, Chavez JA, Roach WG et al (2009) Insulin-stimulated phosphorylation of the Rab GTPase-activating protein TBC1D1 regulates GLUT4 translocation. J Biol Chem 284:30016-30023

44. Cheng KK, Zhu W, Chen B et al (2014) The adaptor protein APPL2 inhibits insulin-stimulated glucose uptake by interacting with TBC1D1 in skeletal muscle. Diabetes. doi:10.2337/db14-0337

45. Funai K, Schweitzer GG, Castorena CM, Kanzaki M, Cartee GD (2010) In vivo exercise followed by in vitro contraction additively elevates subsequent insulin-stimulated glucose transport by rat skeletal muscle. Am J Physiol Endocrinol Metab 298:E999-E1010 
46. An D, Toyoda T, Taylor EB et al (2010) TBC1D1 regulates insulinand contraction-induced glucose transport in mouse skeletal muscle. Diabetes 59:1358-1365

47. Arias EB, Kim J, Funai K, Cartee GD (2007) Prior exercise increases phosphorylation of Akt substrate of $160 \mathrm{kDa}$ (AS160) in rat skeletal muscle. Am J Physiol Endocrinol Metab 292:E1191-E1200

48. Schweitzer GG, Arias EB, Cartee GD (2012) Sustained postexercise increases in AS160 Thr642 and Ser588 phosphorylation in skeletal muscle without sustained increases in kinase phosphorylation. J Appl Physiol 113:1852-1861

49. Funai K, Schweitzer GG, Sharma N, Kanzaki M, Cartee GD (2009) Increased AS160 phosphorylation, but not TBC1D1 phosphorylation, with increased postexercise insulin sensitivity in rat skeletal muscle. Am J Physiol Endocrinol Metab 297:E242-E251

50. Castorena CM, Arias EB, Sharma N, Cartee GD (2014) Postexercise improvement in insulin-stimulated glucose uptake occurs concomitant with greater AS160 phosphorylation in muscle from normal and insulin resistant rats. Diabetes 63:2297-2308

51. Treebak JT, Birk JB, Rose AJ, Kiens B, Richter EA, Wojtaszewski JF (2007) AS160 phosphorylation is associated with activation of $\alpha_{2} \beta_{2} \gamma_{1}$-but not $\alpha_{2} \beta_{2} \gamma 3$-AMPK trimeric complex in skeletal muscle during exercise in humans. Am J Physiol Endocrinol Metab 292:E715-E722

52. Howlett KF, Mathews A, Garnham A, Sakamoto K (2008) The effect of exercise and insulin on AS160 phosphorylation and 14-3-3 binding capacity in human skeletal muscle. Am J Physiol Endocrinol Metab 294:E401-E407

53. Treebak JT, Taylor EB, Witczak CA et al (2010) Identification of a novel phosphorylation site on TBC1D4 regulated by AMP-activated protein kinase in skeletal muscle. Am J Physiol Cell Physiol 298: C377-C385

54. Treebak JT, Pehmøller C, Kristensen JM et al (2014) Acute exercise and physiological insulin induce distinct phosphorylation signatures on TBC1D1 and TBC1D4 proteins in human skeletal muscle. J Physiol 592:351-375

55. Jessen N, An D, Lihn AS et al (2011) Exercise increases TBC1D1 phosphorylation in human skeletal muscle. Am J Physiol Endocrinol Metab 301:E164-E171

56. Breen L, Philp A, Shaw CS, Jeukendrup AE, Baar K, Tipton KD (2011) Beneficial effects of resistance exercise on glycemic control are not further improved by protein ingestion. PLoS One 6:e20613

57. Howlett KF, Sakamoto K, Garnham A, Cameron-Smith D, Hargreaves M (2007) Resistance exercise and insulin regulate AS160 and interaction with 14-3-3 in human skeletal muscle. Diabetes 56:1608-1614

58. Dreyer HC, Drummond MJ, Glynn EL et al (2008) Resistance exercise increases human skeletal muscle AS160/TBC1D4 phosphorylation in association with enhanced leg glucose uptake during postexercise recovery. J Appl Physiol 105:1967-1974

59. Guerra B, Guadalupe-Grau A, Fuentes T et al (2010) SIRT1, AMP-activated protein kinase phosphorylation and downstream kinases in response to a single bout of sprint exercise: influence of glucose ingestion. Eur J Appl Physiol 109: 731-743

60. Levinger I, Jerums G, Stepto NK et al (2014) The effect of acute exercise on undercarboxylated osteocalcin and insulin sensitivity in obese men. J Bone Miner Res Off J Am Soc Bone Miner Res. doi:10.1002/jbmr.2285

61. Ducommun S, Wang HY, Sakamoto K, MacKintosh C, Chen S (2012) Thr649Ala-AS160 knock-in mutation does not impair contraction/AICAR-induced glucose transport in mouse muscle. Am J Physiol Endocrinol Metab 302:E1036-E1043

62. Funai K, Cartee GD (2009) Inhibition of contraction-stimulated AMP-activated protein kinase inhibits contraction-stimulated increases in PAS-TBC1D1 and glucose transport without altering PAS-AS160 in rat skeletal muscle. Diabetes 58:1096-1104
63. Frøsig C, Rose AJ, Treebak JT, Kiens B, Richter EA, Wojtaszewski JF (2007) Effects of endurance exercise training on insulin signaling in human skeletal muscle: interactions at the level of phosphatidylinositol 3-kinase, Akt, and AS160. Diabetes 56:2093-2102

64. Kramer HF, Taylor EB, Witczak CA, Fujii N, Hirshman MF, Goodyear LJ (2007) Calmodulin-binding domain of AS160 regulates contraction- but not insulin-stimulated glucose uptake in skeletal muscle. Diabetes 56:2854-2862

65. Hinkley JM, Ferey JL, Brault JJ, Smith CA, Gilliam LA, Witczak CA (2014) Constitutively active CaMKKalpha stimulates skeletal muscle glucose uptake in insulin-resistant mice in vivo. Diabetes 63:142-151

66. Treebak JT, Glund S, Deshmukh A et al (2006) AMPK-mediated AS160 phosphorylation in skeletal muscle is dependent on AMPK catalytic and regulatory subunits. Diabetes 55:2051-2058

67. Sakamoto K, Hirshman MF, Aschenbach WG, Goodyear LJ (2002) Contraction regulation of Akt in rat skeletal muscle. J Biol Chem 277:11910-11917

68. Sakamoto K, Aschenbach WG, Hirshman MF, Goodyear LJ (2003) Akt signaling in skeletal muscle: regulation by exercise and passive stretch. Am J Physiol Endocrinol Metab 285:E1081-E1088

69. Sakamoto K, Arnolds DE, Fujii N, Kramer HF, Hirshman MF, Goodyear LJ (2006) Role of Akt2 in contractionstimulated cell signaling and glucose uptake in skeletal muscle. Am J Physiol Endocrinol Metab 291:E1031-E1037

70. Goodyear LJ, Chang PY, Sherwood DJ, Dufresne SD, Moller DE (1996) Effects of exercise and insulin on mitogen-activated protein kinase signaling pathways in rat skeletal muscle. Am J Physiol 271: E403-E408

71. Lund S, Pryor PR, Ostergaard S, Schmitz O, Pedersen O, Holman GD (1998) Evidence against protein kinase B as a mediator of contraction-induced glucose transport and GLUT4 translocation in rat skeletal muscle. FEBS Lett 425:472-474

72. Brozinick JT Jr, Birnbaum MJ (1998) Insulin, but not contraction, activates Akt/PKB in isolated rat skeletal muscle. J Biol Chem 273: 14679-14682

73. Sakamoto K, Arnolds DE, Ekberg I, Thorell A, Goodyear LJ (2004) Exercise regulates Akt and glycogen synthase kinase-3 activities in human skeletal muscle. Biochem Biophys Res Commun 319:419 425

74. Markuns JF, Wojtaszewski JF, Goodyear LJ (1999) Insulin and exercise decrease glycogen synthase kinase-3 activity by different mechanisms in rat skeletal muscle. J Biol Chem 274:24896-24900

75. Wojtaszewski JF, Higaki Y, Hirshman MF et al (1999) Exercise modulates postreceptor insulin signaling and glucose transport in muscle-specific insulin receptor knockout mice. J Clin Invest 104: 1257-1264

76. Widegren U, Jiang XJ, Krook A et al (1998) Divergent effects of exercise on metabolic and mitogenic signaling pathways in human skeletal muscle. FASEB J 12:1379-1389

77. Mu J, Brozinick JT Jr, Valladares O, Bucan M, Birnbaum MJ (2001) A role for AMP-activated protein kinase in contraction- and hypoxia-regulated glucose transport in skeletal muscle. Mol Cell 7:1085-1094

78. Jorgensen SB, Viollet B, Andreelli F et al (2004) Knockout of the alpha2 but not alpha1 5'-AMP-activated protein kinase isoform abolishes 5-aminoimidazole-4-carboxamide-1- $\beta$ - 4 ribofuranosidebut not contraction-induced glucose uptake in skeletal muscle. J Biol Chem 279:1070-1079

79. O'Neill HM, Maarbjerg SJ, Crane JD et al (2011) AMP-activated protein kinase (AMPK) $\beta 1 \beta 2$ muscle null mice reveal an essential role for AMPK in maintaining mitochondrial content and glucose uptake during exercise. Proc Natl Acad Sci U S A 108:16092-16097

80. Frøsig C, Pehmøller C, Birk JB, Richter EA, Wojtaszewski JF (2010) Exercise-induced TBC1D1 Ser237 phosphorylation and 14-3-3 protein binding capacity in human skeletal muscle. J Physiol 588:4539-4548 
81. Vendelbo MH, Moller AB, Treebak JT et al (2014) Sustained AS160 and TBC1D1 phosphorylations in human skeletal muscle 30 minutes after a single bout of exercise. J Appl Physiol 117:289-296

82. Cartee GD, Briggs-Tung C, Kietzke EW (1993) Persistent effects of exercise on skeletal muscle glucose transport across the life-span of rats. J Appl Physiol 75:972-978

83. Wojtaszewski JF, Hansen BF, Gade et al (2000) Insulin signaling and insulin sensitivity after exercise in human skeletal muscle. Diabetes 49:325-331

84. Hansen PA, Nolte LA, Chen MM, Holloszy JO (1998) Increased GLUT-4 translocation mediates enhanced insulin sensitivity of muscle glucose transport after exercise. J Appl Physiol 85:1218-1222

85. Howlett KF, Sakamoto K, Hirshman MF et al (2002) Insulin signaling after exercise in insulin receptor substrate-2-deficient mice. Diabetes 51:479-483

86. Treebak JT, Frøsig C, Pehmøller C et al (2009) Potential role of TBC1D4 in enhanced post-exercise insulin action in human skeletal muscle. Diabetologia 52:891-900

87. Ikeda S, Tamura Y, Kakehi S et al (2013) Exercise-induced enhancement of insulin sensitivity is associated with accumulation of M2-polarized macrophages in mouse skeletal muscle. Biochem Biophys Res Commun 441:36-41

88. Consitt LA, Van Meter J, Newton CA et al (2013) Impairments in site-specific AS160 phosphorylation and effects of exercise training. Diabetes 62:3437-3447

89. Xie X, Chen Y, Xue P et al (2009) RUVBL2, a novel AS160-binding protein, regulates insulin-stimulated GLUT4 translocation. Cell Res 19:1090-1097

90. Ren W, Cheema S, Du K (2012) The association of ClipR-59 protein with AS160 modulates AS160 protein phosphorylation and adipocyte Glut4 protein membrane translocation. J Biol Chem 287:26890-26900

91. Ho PC, Lin YW, Tsui YC, Gupta P, Wei LN (2009) A negative regulatory pathway of GLUT4 trafficking in adipocyte: new function of RIP140 in the cytoplasm via AS160. Cell Metab 10:516-523

92. Cao S, Li B, Yi X et al (2012) Effects of exercise on AMPK signaling and downstream components to PI3K in rat with type 2 diabetes. PLoS One 7:e51709

93. Xiao Y, Sharma N, Arias EB, Castorena CM, Cartee GD (2013) A persistent increase in insulin-stimulated glucose uptake by both fasttwitch and slow-twitch skeletal muscles after a single exercise session by old rats. Age (Dordr) 35:573-582

94. Vind BF, Pehmøller C, Treebak JT et al (2011) Impaired insulininduced site-specific phosphorylation of TBC1 domain family, member 4 (TBC1D4) in skeletal muscle of type 2 diabetes patients is restored by endurance exercise-training. Diabetologia 54:157-167

95. Brandt N, De Bock K, Richter EA, Hespel P (2010) Cafeteria dietinduced insulin resistance is not associated with decreased insulin signaling or AMPK activity and is alleviated by physical training in rats. Am J Physiol Endocrinol Metab 299:E215-E224

96. Chadt A, Leicht K, Deshmukh A et al (2008) Tbc1d1 mutation in lean mouse strain confers leanness and protects from diet-induced obesity. Nat Genet 40:1354-1359

97. Szekeres F, Chadt A, Tom RZ et al (2012) The Rab-GTPaseactivating protein TBC1D1 regulates skeletal muscle glucose metabolism. Am J Physiol Endocrinol Metab 303:E524-E533

98. Dokas J, Chadt A, Nolden T et al (2013) Conventional knockout of Tbc1d1 in mice impairs insulin- and AICAR-stimulated glucose uptake in skeletal muscle. Endocrinology 154:3502-3514

99. Lansey MN, Walker NN, Hargett SR, Stevens JR, Keller SR (2012) Deletion of Rab GAP AS160 modifies glucose uptake and GLUT4 translocation in primary skeletal muscles and adipocytes and impairs glucose homeostasis. Am J Physiol Endocrinol Metab 303: E1273-E1286

100. Wang HY, Ducommun S, Quan C et al (2013) AS160 deficiency causes whole-body insulin resistance via composite effects in multiple tissues. Biochem J 449:479-489 